\title{
Politische Grundlagen und Folgen von Dualisierungsprozessen: Eine politi- sche Ökonomie der Hartz-Reformen
}

\author{
Zweifellos stellen die Hartz-Reformen einen tief greifenden Einschnitt in der deutschen \\ Arbeitsmarkt- und Sozialpolitik dar. Ihre sozio-ökonomischen Ursachen und Wirkungen \\ wurden bereits vielfach untersucht und beschrieben. Dieser Beitrag lenkt hingegen die \\ Aufmerksamkeit einerseits auf die gesellschaftlichen Voraussetzungen, die den arbeits- \\ markt- und sozialpolitischen Wandel ermöglicht haben, andererseits auf die Folgen, die \\ sich daraus für die politischen Einstellungs- und Verhaltensmuster von Arbeitsmarktteil- \\ nehmern ergeben. Die Hartz-Reformen werden als Teil eines breiteren Dualisierungs- \\ prozesses begriffen, der auch in anderen europäischen Ländern beobachtet wird.
}

URSULA DALLINGER, SEBASTIAN FÜCKEL

\section{Einleitung}

Seit wenigstens zwei Jahrzehnten lassen sich in einer Vielzahl europäischer Länder einschneidende Veränderungen in der jeweiligen Arbeitsmarkt- und Sozialpolitik beobachten, die als Deregulierung, Dualisierung, Flexibilisierung oder Liberalisierung beschrieben werden. Zweifellos sind auch die Hartz-Reformen Bestandteil dieser Wandlungsprozesse. Sie beinhalten arbeitsmarkt- und sozialpolitische Instrumente, die einerseits die soziale Sicherung Arbeitsloser senken und den Druck auf diese erhöhen, dem Arbeitsmarkt auch bei schlechteren Bedingungen zur Verfügung zu stehen (Kommodifizierung) und andererseits den Unternehmen neue, flexiblere Beschäftigungsformen bieten (Deregulierung). Zentral ist, dass diese arbeitsmarkt- und sozialpolitischen Reformen primär sogenannte Outsider treffen, wie z.B. Arbeitslose, in den Arbeitsmarkt erneut zurückkehrende Frauen oder Berufsanfänger, denen der Wiedereintritt in den Arbeitsmarkt nur zu den neuen Konditionen gelingt. Während sogenannte Insider weiter reguläre, relativ gut gesicherte Jobs innehaben mit kollektiv verhandelten Löhnen und gesetzlichem Kündigungsschutz, tragen Outsider des Arbeitsmarktes die Kosten der Reformen und erfahren so letztlich eine Ungleichbehandlung im Hinblick auf soziale Sicherung bei Arbeitslosigkeit wie auch im Hinblick auf die Chance, ein Arbeitsverhältnis mit ausreichendem Lohn und sozialer Sicherung zu erhalten.
Der vorliegende Beitrag vertieft die These der Dualisierungsprozesse, indem nicht nur seine institutionelle Dimension (also der Politikwandel) betrachtet, sondern der Blick auf dessen polit-ökonomische Grundlagen und Auswirkungen ausgeweitet wird. Wir zeigen, dass sich bereits längerfristig die Einkommensverteilung in einer Weise wandelte, die die Wohlfahrtsposition mittlerer und unterer Einkommensschichten polarisierte und so auch gespaltene politische Interessen in der Bevölkerung förderte. Diese Spaltung war eine Voraussetzung für eine Arbeitsmarkt- und Sozialpolitik, die selektiv die Kosten der Flexibilisierung und De-Kommodifizierung Outsidern zuweist. Wir gehen weiter davon aus, dass institutionelle Dualisierung erst durch die Verschiebung der politisch-ideologischen Programmatik linker, sozialdemokratischer Parteien möglich wurde, die traditionell als politische Repräsentanten schwächerer Einkommensschichten gelten (Lipset/Rokkan 1967), sich nun aber verstärkt an den Interessen der Mittelschichten als wahlentscheidende Gruppen orientieren. Institutionelle Dualisierung mündet weiter - so die hier formulierte Erwartung - in dualisierte politische Einstellungs- und Verhaltensmuster. Arbeitsmarktoutsider, deren soziale Lage sich durch ein hohes Arbeitslosigkeitsrisiko, geringe Einkommenschancen und eine schlechte Integration in die sozialen Sicherungssysteme auszeichnet, sollten sich zunehmend von der Sozialdemokratie abwenden, wie es die These vom „Dilemma sozialdemokratischer Parteien“ begründet (Rueda 2005; Lindvall/Rueda 2012). Diese Annahmen werden primär mit Blick auf Deutschland empirisch 
untersucht, jedoch durch einen Vergleich mit weiteren Ländern - Dänemark, die Niederlande und das Vereinigte Königreich - gerahmt. Diese Länder repräsentieren unterschiedliche arbeitsmarkt- und sozialpolitische Strategien zur Lösung der Arbeitsmarktstrukturprobleme der 1980er und 1990er Jahre (Thelen 2012; Clasen/Clegg in diesem Heft).

Im Folgenden wird das Dualisierungskonzept präzisiert und zu den Hartz-Reformen in Beziehung gesetzt (Abschnitt 2). Die nächsten Abschnitte wenden sich dem Wandel der Einkommensstrukturen (3) sowie der Verschiebung der politisch-ideologischen Ausrichtung sozialdemokratischer Parteien (4) zu, beide Male mit der Absicht, die politökonomischen Grundlagen der späteren engeren institutionellen Dualisierung in Form von Reformen, die Outsider belasten, zu zeigen. Abschnitt 5 fragt nach den Folgen institutioneller Dualisierung für politische Verhaltensweisen bei den In- und Outsidern. Abschließend ziehen wir ein Fazit zur politischen Ökonomie der Dualisierung (6).

\section{Die Hartz-Reformen als Teil von Dualisierungsprozessen}

Der Wandel der Arbeitsmarkt- und Sozialpolitik wird als ein Prozess der Dualisierung diskutiert, da „policies increasingly differentiate rights, entitlements, and services provided to different categories of recipients" (Emmenegger et al. 2012a, S. 10). Dies gilt auch für die Hartz-Reformen in der Bundesrepublik, die „zwei weitgehend getrennte Systeme sozialer Sicherung mit stark differierenden sozialen Rechten" (Dingeldey 2010, S. 18) etablierten, was den Begriff einer institutionellen Dualisierung rechtfertigt. Mehrere Elemente der Politikreformen generieren In- und Outsider des Arbeitsmarktes: So werden Personen bereits bei einer über ein Jahr dauernden Arbeitslosigkeit zu Empfängern von steuerfinanzierter, bedarfsgeprüfter Grundsicherung, was ihre Einkommenslage verschlechtert, verschärfte Regeln der Vermögensanrechnung mit sich bringt und wie eine Sanktion von Arbeitslosigkeit wirkt. Die Zusammenlegung von Arbeitslosen- und Sozialhilfe zum neu geschaffenen Arbeitslosengeld II bringt deutliche Nachteile gegenüber der früheren Versicherungsleistung bzw. der einer Versicherungsleistung nachempfundenen Arbeitslosenhilfe. Darüber hinaus haben die Hartz-Reformen zum Anstieg atypischer (und prekärer) Beschäftigungsverhältnisse zulasten des Normalarbeitsverhältnisses beigetragen (Keller et al. 2012), indem geringfügige und befristete Beschäftigungsverhältnisse sowie mit dem Existenzgründungszuschuss die Ausbreitung von Solo- und Scheinselbstständigkeit weiter befördert wurden (Gießelmann 2009, S. 216ff.; Palier/Thelen 2010). Nun zielen diese Instrumente nicht explizit auf eine sozio-ökonomische Dualisierung, haben aber diese implizit zur Folge.

Als Arbeitsmarktoutsider werden Individuen mit einem besonders hohen Risiko, in atypische Beschäftigung zu mün- den oder arbeitslos zu sein, definiert (Emmenegger et al. 2012b, S. 30). Denn Arbeitslose oder junge Erwachsene, die den Zugang zum Arbeitsmarkt suchen, erhalten diesen $\mathrm{Zu}$ gang mit hoher Wahrscheinlichkeit nur zu den neuen Arbeitsplatzkonditionen und müssen ein niedriges Entlohnungsniveau, geringe oder fehlende Sozialleistungen wie auch fehlenden Kündigungsschutz akzeptieren (Giesecke/Heisig 2010). Als Insider gelten durch das Normalarbeitsverhältnis geschützte Gruppen, die (noch) von den im Rahmen industrieller Beziehungen erzielten Regelungen profitieren, auch wenn die Reichweite tarifvertraglich regulierter Arbeitsverhältnisse und Löhne längst schrumpft. Nach dem Dualisierungskonzept ist die Schaffung von Arbeitsplätzen „zweiter Wahl“ und abgesenkter Leistungen für Arbeitslose das Mittel, um an den Rändern des Arbeitsmarktes Flexibilität für Unternehmen zu schaffen und zugleich die bisherigen Standards der Kernbelegschaft zu bewahren. Allerdings wird kontrovers diskutiert, ob eine dualisierende Arbeitsmarktpolitik allein Outsider trifft, oder nicht vielmehr auf alle Beschäftigten ausstrahlt (siehe Bothfeld/Rosenthal in diesem Heft).

Die arbeitsmarktpolitischen Reformen sind eingebettet in seit Längerem wachsende Einkommensdisparitäten. Diese bringen ebenso dualisierte soziale Strukturen und Dispositionen der Bürger hervor, was institutionellen Reformen die Basis bot.

\section{Einkommensungleichheit und Dualisierung}

Mit dem internationalen Wettbewerb und internationaler Lohnkonkurrenz gingen gering produktive Jobs in Bereichen mit hohem Rationalisierungsdruck verloren, die vorher relativ gut entlohnt waren und oft Familienernährerlöhne boten. Diese Arbeitsplätze für Angelernte in der Industrie wurden teils automatisiert, teils outgesourct. Deutschland entwickelte sich wie andere Länder zu einer Dienstleistungsgesellschaft (Esping-Andersen 2009). Es besteht weitgehend Konsens in der Forschung, dass sich in Dienstleistungsgesellschaften die Einkommensdisparitäten durch ein ganzes Bündel an Ursachen vertiefen: vermehrte Nachfrage nach Höherqualifizierten, Erosion der Tarifbindung und kollektiver Lohnverhandlungen, Entstehung deregulierter Arbeit, Ausbreitung des Niedriglohnsektors (Harrison/Bluestone 1988; Lohmann 2010; Bispinck/Bosch 2008). Insgesamt verschlechterten sich die Arbeitsmarkt- und Einkommenschancen der geringer Qualifizierten in unteren Lagen der Sozialhierarchie, während sich Arbeitsmarkt- und Einkommenschancen für besser Qualifizierte noch weiter verbesserten. Zeitraum und Umfang der Verschiebung von Beschäftigung in den Dienstleistungssektor mitsamt ihren Folgen für die Einkommensverteilung variieren zwischen postindustriellen Ländern, auch abhängig vom Arbeitsrecht, dem System kollektiver Lohnverhandlungen oder dem jeweiligen Produktionsregime. 
ABB. 1

\section{Anteil der Bevölkerungsquintile am gesamten Markteinkommen}

Angaben in Prozent

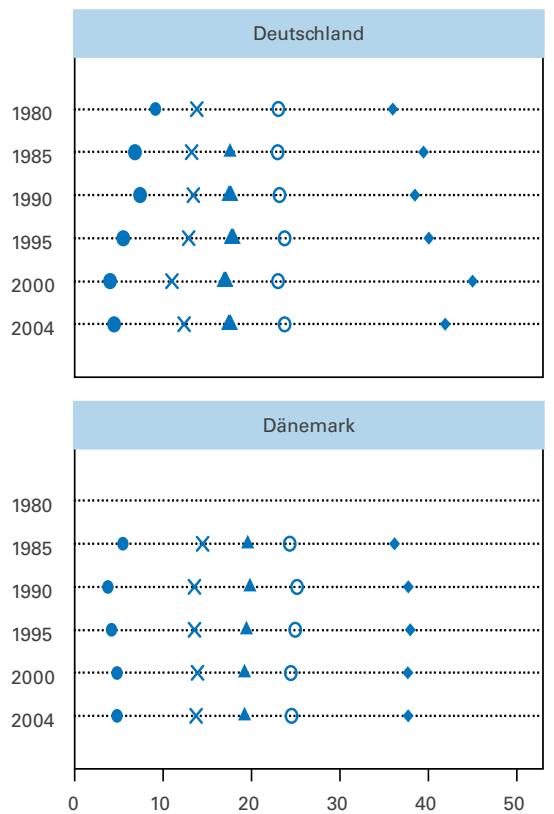

kommen erkennen kann. Dieser Maßzahl liegt der Gedanke zugrunde, dass Disproportionalität - wenn also ein Fünftel der Bevölkerung weniger als 20 \% der Einkommen erhält Ungleichheit anzeigt: Auch der konkrete Prozentsatz der gesamten Markteinkommen, der an einzelne Quintile fließt, ist informativ im Hinblick auf die relative Einkommensposition einzelner Gruppen. Dieses Instrument legt lediglich Einkommen zugrunde, bietet also keine auf der Basis von zusätzlichen Merkmalen wie Berufsprestige oder Entscheidungsbefugnisse gebildete Klassifizierung, wie Modelle für soziale Schichten oder Klassen. Dennoch sprechen wir im Folgenden von sozialen Schichten bzw. Klassen, da auch die relative Einkommensposition eine gute Annäherung an soziale Hierarchie darstellt, weil der Zugang zu weiteren Ressourcen und Lebenschancen durch Einkommen strukturiert ist. ${ }^{1}$

Der Anteil des Gesamteinkommens, der an Haushalte mit geringem Einkommen fließt, sank - abgesehen von Dänemark. Besonders deutlich war der Rückgang in Deutschland (von 9,1\% 1980 auf 4,5\% 2004) und Großbritannien (von $5 \% 1986$ auf 3,9 \% 2004). ${ }^{2}$ In beiden Ländern waren dagegen den obersten Einkommensgruppen Zugewinne möglich.

Wenn man die sozialstrukturellen Grundlagen erkundet, die die Basis einer Arbeitsmarkt- und Sozialpolitik mit dualisierenden Effekten war, sind aber weniger die Abstände zwischen oben und unten wichtig, sondern für mögliche politische Allianzen kommt es eher auf ähnliche Erfahrungen in Mittel- und Unterschicht bei den Einkommenschancen an, die dann ähnliche politische Interessen begründen können. Nach Abbildung 1 verschlechterte sich die Einkommensposition der Haushalte im mittleren Bereich (drittes Quintil) aber nur gering oder gar nicht. Lediglich in Großbritannien verzeichneten auch mittlere Einkommen keine stabile Einkommensposition, sondern ihr Anteil sank. Allenfalls die untere Mittelschicht (zweites Quintil) macht die Verluste der untersten Einkommensgruppe mit und hat somit potenziell ähnliche Interessen im Hinblick auf Arbeitsmarkt- und Sozialpolitik. Aus dieser Entwicklung lässt sich folgern, dass mittlere und obere Mittelschicht weiter stabile Einkommenschancen haben. Für sie bot der Arbeitsmarkt weiter Potenziale, weniger dagegen für Unqualifizierte, Berufsanfänger und Frauen mit eingeschränkter zeitlicher Verfügbarkeit (Häusermann/Schwander 2012). Es sind diese Gruppen, die zu atypischer, gering entlohnter Arbeit greifen müssen. Die - außer in Großbritannien - stabile Lage der mittleren Schicht
1 Unsere Maßzahl fängtTrends der Verteilungsstrukturen einer Gesellschaft ein, aber keine Einkommensmobilität von Einzelnen oder Haushalten, da es sich nicht um Paneldaten zu Individuen handelt. Dies ist aber unproblematisch, da soziale Strukturen ohnehin als das von Individuen unabhängige Schichtungs- oder Ungleichheitsgefüge von Gesellschaften definiert werden.

2 Die Ungleichheitsentwicklung in Deutschland war ab der "Wiedervereinigung" 1991 durch die hohe Arbeitslosigkeit in den neuen Bundesländern mit geprägt. Diese Probleme gehen in die Abbildung und den gezeigten Rückgang des Anteils des untersten Bevölkerungsfünftels am Markteinkommen zwischen 1990 und 1995 ein. Aber bereits vor der Wiedervereinigung wuchs noch ohne ostdeutschen Beitrag die Ungleichheit in Westdeutschland deutlich von einem Wert in Höhe von $30.3 \mathrm{im}$ Jahr 1981, über $36.1 \mathrm{im}$ Jahr 1994 auf einen Wert von 38.8 im Jahr 2004. Allerdings ist der Anstieg des GINI-Koeffizienten (ein weiteres Ungleichheitsmaß) deutlicher, wenn man die Beitrittsländer mit einbezieht. Dann steigt der GINI von 36.9 im Jahr 1994 auf $40.2 \mathrm{im}$ Jahr 2004 (Immervoll/Richardson 2013, S. 20). 
bestätigt, dass besonders die relative Einkommensposition der Mitte geschützt ist. Während es in den Niederlanden und Dänemark mehr oder weniger gelingt, auch Haushalte mit geringen Einkommen zu stützen, konserviert Deutschlands korporatistisches Modell industrieller Beziehungen die Position der mittleren, weniger aber der unteren Einkommensklassen. So sollten sich besonders in Deutschland Grundlagen für dualisierende Politikreformen entwickelt haben.

Diese für die Mittelschicht relativ geringere Bedrohung durch Einkommensverluste bietet kaum die sozialstrukturelle Grundlage für Koalitionen mit denen am unteren Ende der Einkommenshierarchie. Im Gegenteil: Die Kluft zwischen gesicherten Mittelschichteinkommen und Haushalten mit den geringsten Markteinkommen wächst und somit die Möglichkeit für die Politik, eine die Dualisierung institutionell absichernde Arbeitsmarktreform ohne Widerstand der Mittelschichtwähler durchzuführen. Die Mitte benötigt kompensatorische soziale Sicherung bei Arbeitslosigkeit nicht nur weniger als die Unterschichten mit einem weitaus größeren Arbeitslosigkeitsrisiko. Da Sozialleistungen für Arbeitslose und somit hohe Lohnnebenkosten sogar als Ursache fehlender Jobs gelten, sieht die qualifizierte Mitte diese finanzielle Belastungen bringenden Leistungen eher skeptisch, da Insider diese Leistungen finanzieren, aber Outsider sie brauchen. Nur überproportional von Arbeitslosigkeit Betroffene, das sind vornehmlich Geringqualifizierte, befürworten den Ausbau der Sozialen Sicherung für Arbeitslose (Giger et al. 2012; Dallinger 2013). In- und Outsider haben unterschiedliche arbeitsmarktpolitische Präferenzen (Rueda 2005; Marx 2012). Sozialpolitische Präferenzen der qualifizierten Arbeiter- und Angestelltenmittelschicht entfernen sich zunehmend von denen der Unqualifizierten.

Das muss nicht so bleiben. Denn die Mitte ist von den Arbeitsreformen zumindest indirekt betroffen, was sich in der Mitte als Angst vor einem Arbeitsplatzverlust manifestiert, der heute fatalere Folgen hat als vor den Hartz-Reformen. Das höhere Risiko des Abstiegs erzeugt subjektive Ängste bei der Mitte, die erstaunlicherweise wenig mit objektiver Beschäftigungsunsicherheit zusammenhängen (Lengfeld/Hirschle 2009; Erlinghagen 2010).

\section{Die Ausrichtung der Sozialdemo- kratie an der politischen Mitte}

Wählerinteressen werden nach dem klassischen demokratietheoretischen Modell durch Parteien aufgegriffen, in die Politik transportiert und beeinflussen so Regierungspolitik (Downs 1957). Parteien sollten bestimmte soziale Gruppen und deren Anliegen repräsentieren, sozialdemokratische Parteien eben die Arbeiter- und Unterschichtinteressen. An dieser klaren Zuordnung zwischen „Klasseninteressen“ und (hier sozialdemokratischen) Parteien sind Abstriche zu machen, da sich Arbeiter auch mit christdemokratischen Par- teien identifizieren. Zudem wird eine Bewegung der Sozialdemokratie weg von „Arbeiterinteressen“ in die Mitte des politischen Spektrums beobachtet (Pontusson 1995; Nelson/ Way 2007; Rueda 2005). Da mit dem Übergang zur Dienstleistungsgesellschaft der Anteil der Arbeiter schrumpft, passt die Sozialdemokratie sich an das Wählerspektrum der postindustriellen Gesellschaft an und sucht verstärkt politische Unterstützung in den Mittelschichten. Die Literatur zur Dualisierung der Arbeitsmarktpolitik beobachtet zudem eine Verschiebung der Zielgruppe sozialdemokratischer Parteien hin zu im Arbeitsmarkt relativ gut etablierten Facharbeitern und Angestellten, was für die politischen Programme, die die Sozialdemokratie Wählern anbietet, Folgen hat. Mit der Ausrichtung an den Interessen beruflich etablierter Insider habe sich eine konservativere Parteiprogrammatik entwickelt. Die sozialdemokratischen Parteien in vielen Ländern rückten ins politische Zentrum und die Allianz zwischen Unter- und Mittelschicht wurde zunehmend aufgegeben (Iversen/Soskice 2009).

Wie sich die ideologische Links-Rechts-Position der Sozialdemokratie entwickelte, lässt sich mit Daten des Comparative Party Manifesto Project (CMP) empirisch beobachten. Hier werden ideologische Positionen von Parteien aus deren Wahlprogrammen textanalytisch herausgefiltert und in einen Links-Rechts-Index transformiert. ${ }^{3}$ Abbildung 2 verwendet eine Variante des Links-Rechts-Index von Kim/Fording (1998), die zudem einen Index zur Links-Rechts-Position des Medianwählers ${ }^{4}$ zur Verfügung stellen. So kann die Bewegung der Sozialdemokratie hin zur Mitte, wo Mehrheiten vermutet werden, gezeigt werden.

Betrachtet man die Verschiebung der politisch-ideologischen Orientierung der Sozialdemokratie, dann zeigt sich für Deutschland zunächst sogar bis 1990 eine Bewegung weiter nach links. Ab ca. 1990 aber sank der Indikator in die Mitte des Links-Rechts-Spektrums. Damit erreicht die Sozialdemokratie auch die Linie für den Medianwähler, entspricht also nun Mehrheitspräferenzen, die sie in den 1970er und 1980er Jahren mit einer weiter links liegenden Position im politischen Spektrum verfehlt hatte (Pontusson/Rueda 2010). In den Niederlanden verschob sich bereits ab 1977 die ideologische Position der Sozialdemokratie deutlich in die Mitte, in Großbritannien und Dänemark ebenfalls ab Beginn der 1990er Jahre. In allen Ländern war damit eine Annäherung an den Medianwähler verbunden, die Regierungsmacht und somit Einfluss auf faktische Politik vermittelte. Abbildung 2 legt nahe, dass die Sozialdemokratie in die Mitte des politischen Spektrums rückt und dann arbeitsmarktpolitische Reformen umsetzt, die die Zielgruppe in den mitt-

3 Die Daten zur ideologischen Position der Parteien des Party Manifesto Projects basieren auf quantitativen Inhaltsanalysen von Wahlprogrammen.

4 Der Begriff des Medianwählers folgt dem Gedanken, dass um die Mitte einer Verteilung häufig die meisten Stimmen liegen werden. 
ABB. 2

\section{Links-Rechts-Position sozialdemokratischer Parteien und des Medianwählers} $100=$ links, 0 = rechts
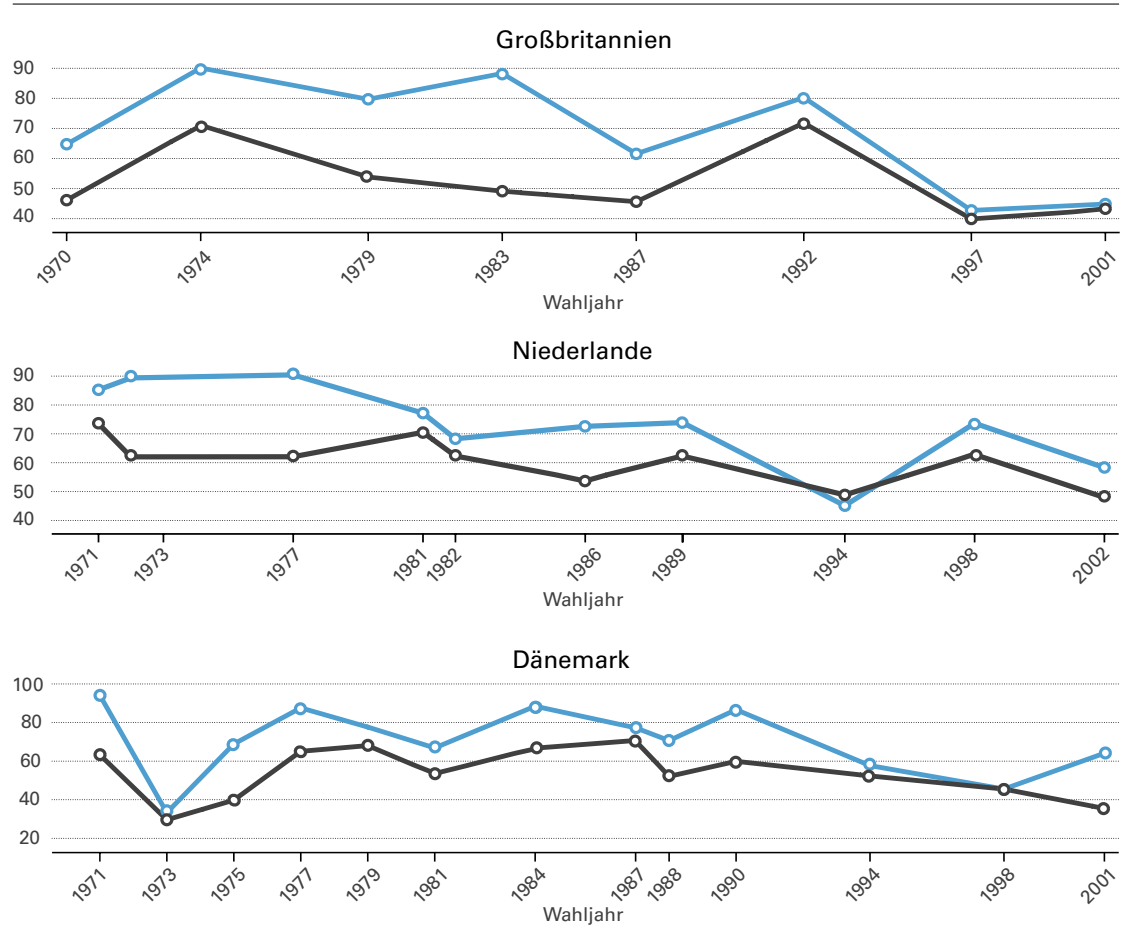

Deutschland

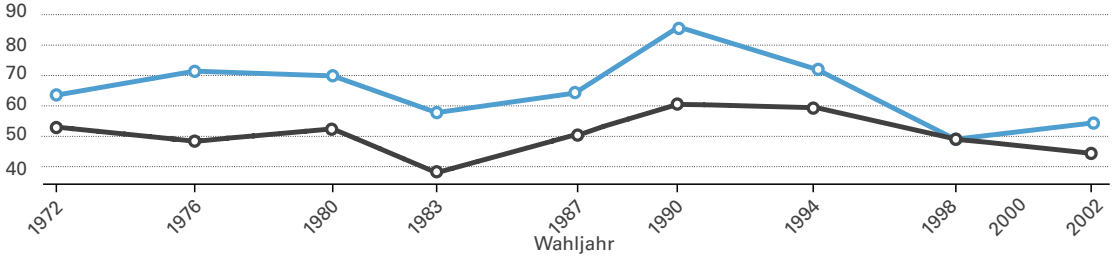

Anmerkung: Sozialdemokratische Parteien: DK = Socialdemokraterne (SD), $\mathrm{DE}=$ Sozialdemokratische Partei Deutschlands (SPD), NL = Partij Van De Arbeid (PvdA) GB $=$ Labour Party (Labour).

Quelle: Darstellung der Autoren auf Basis von Daten aus Kim/Fording (2003).

leren Schichten von negativen Folgen arbeitsmarktpolitischer Reformen zugunsten der immer wieder angemahnten größeren Flexibilität von Arbeit ausspart. Diese Feststellung ist weder ein Urteil über den arbeitsmarktpolitischen Sinn der Maßnahmen, noch negiert sie, dass subjektiv auch mittlere Wählerschichten verunsichert sind. Es soll lediglich der Dualisierungsprozess empirisch verdeutlicht werden.

Vor dem Hintergrund der ins politische Zentrum gerückten Sozialdemokratie wird auch plausibel, dass man inzwischen keine Beziehung mehr zwischen dem Einfluss linker Parteien in der Regierung und dem Umfang an Lohnungleichheit findet. Andere international vergleichenden Studien zum Zusammenhang zwischen Ungleichheit und politischen Reaktionen fanden, dass nur bei einer starken Mobilisierung unterprivilegierter Wähler die Sozialdemokratie mit sozialund arbeitsmarktpolitischen Programmen reagiert, die Ein- kommensdiskrepanzen verringern, da eine hohe Wahlbeteiligung in der Regel untere Schichten an die Wahlurne bringt (Pontusson et al. 2002; Pontusson/Rueda 2010). Die Sozialdemokratie mag künftig - bei entsprechender Wählermobilisierung - wieder nach links rücken, weil die sozialen Folgen der Deregulierung sichtbar werden. Gleichwohl ist die Sozialdemokratie in einer schwierigen Position: Einerseits wandern Arbeitsmarktinsider in ökonomisch unbequemen Phasen zu den Mitte-Rechts-Parteien ab, andererseits die Arbeitsmarktoutsider zu linken Parteien. Gleich, ob die Sozialdemokratie Dualisierung verhindert oder fördert, sie befindet sich in einem Dilemma, da bei jeder Option ein Teil der potenziellen Wähler entfremdet wird (Emmenegger et al. 2012b).

\section{Politische Verhaltensweisen von Arbeitsmarktinsidern und -outsidern}

Was folgt aus der institutionellen Dualisierung für das politische Verhalten von Arbeitsmarktinsidern und -outsidern? Die politische Einstellungs- und Verhaltensforschung konnte den Einfluss der individuellen materiellen Lage und des damit einhergehenden sozialen Status auf politische Präferenzen und Verhaltensweisen in der Vergangenheit immer wieder bestätigen (z. B. Verba et al. 1995; Schoen 2005). Da institutionelle Dualisierung, wie oben erläutert, zu einer Verschärfung der sozialen Ungleichheit zwischen Insidern und Outsidern (soziale Sicherung, Arbeitsmarktstatus) beiträgt, ist zu erwarten, dass sich auch die politischen Präferenzen zwischen beiden Gruppen im Anschluss an den arbeitsmarktund sozialpolitischen Wandel auseinanderentwickelten.

Dabei erscheint die politische Orientierung gegenüber sozialdemokratischen Parteien besonders interessant, denn zum einen hat sich die Sozialdemokratie im Zuge ihrer inhaltlichen Neuausrichtung zunehmend von den Interessen unterer sozialer Schichten, die sowohl ihre klassische Kernklientel (Lipset/Rokkan 1967) als auch überdurchschnittlich viele Outsider beherbergen (Häusermann/Schwander 2012, S. 36ff.), distanziert. Zum anderen illustriert Übersicht 1, dass wesentliche arbeitsmarkt- und sozialpolitische Reformen, die vornehmlich Arbeitsmarktoutsider belasten, von Regierungen umgesetzt wurden, an denen sozialdemokratische Parteien beteiligt waren. Daher sollten vor allem Arbeitsmarktoutsider sozialdemokratischen Parteien ihre politische Unterstützung im Anschluss an die arbeitsmarkt- und sozialpolitischen Reformen verweigert haben.

Nachfolgend soll diese These einem Test unterzogen werden, indem empirische Befunde zur Entwicklung des Wahlverhaltens von Arbeitsmarktinsidern und -outsidern gegenübergestellt werden. Als Datengrundlage werden die ersten fünf Wellen des European Social Survey (ESS) für Dänemark, Deutschland und das Vereinigte Königreich herangezogen. Da die ESS-Wellen leider nur den Zeitraum nach der Jahrtausendwende abdecken, institutionelle Dualisierungsmaß- 
- Verkürzung der Bezugsperiode von Geldleistungen für Arbeitslose auf sieben Jahre

- Verschärfung der Bezugskriterien von Geldleistungen für Arbeitslose (zuvor mindestens sechs Monate in regulärer Beschäftigung)

- Verpflichtung zurTeilnahme an Aktivierungsmaßnahmen nach vier Jahren

-Wiedereingliederungsplan für Langzeitarbeitslose (individuell verpflichtend)

1995/96 Arbeitsmarktreformpaket II

- Verkürzung der Bezugsperiode von Geldleistungen für Arbeitslose auf fünf Jahre

- Verschärfung der Bezugskriterien von Geldleistungen für Arbeitslose (mindestens 52 Wochen in regulärer Beschäftigung innerhalb von drei Jahren)

- Verschärfung der Bezugskriterien von Geldleistungen für unter 25-Jährige (Kürzung der Geldleistungen und Verpflichtung zurTeilnahme an Weiterbildungsmaßnahmen nach 26 Wochen)

- Verpflichtung zurTeilnahme an Aktivierungsmaßnahmen nach zwei Jahren

- Verschärfung der Sanktionen bei Ablehnung eines Job-Angebots

1997/98 Gesetz zur aktiven Sozialpolitik
- Verpflichtung zurTeilnahme an Aktivierungsmaßnahmen für Bezieher von Sozialhilfe

- Verkürzung der Bezugsperiode von Geldleistungen für Arbeitslose auf vier Jahre

- Aufhebung der verlängerten Bezugsperiode von Geldleistungen für 50- bis 54-Jährige

- Verpflichtung zurTeilnahme an Aktivierungsmaßnahmen nach einem Jahr

- Verschärfung der Zumutbarkeitskriterien zur Arbeitsaufnahme

- Reform des Frühverrentungssystems

Deutschland (Sozialdemokratische Partei Deutschlands 1998-2005)
Abschaffung der originären Arbeitslosenhilfe

2002 Job-AQTIV-Gesetz

2003/05 Hartz-Gesetze
- Abschaffung der Arbeitslosenhilfe für Arbeitslose, die zuvor keinen Anspruch auf Arbeitslosengeld hatten

- Implementierung von Regelungen zur staatlichen Förderung befristeter Vertretungsstellen

- Lockerung der Bedingungen für Leih-/Zeitarbeit

- Erweiterung zumutbarerTätigkeiten für Arbeitslose um Zeitarbeit

- Verschärfung der Sanktionen (Leistungskürzungen) bei unkooperativen Verhalten Arbeitsloser

- Absenkung der Freibeträge für Empfänger von Arbeitslosenhilfe

- Lockerung der Voraussetzungen zur Schaffung geringfügiger und befristeter Beschäftigungsverhältnisse

- Einführung des Existenzgründungszuschuss

- Einführung des Arbeitslosengeldes I und II (vgl. übrige Beiträge des Sammelbandes)

Vereinigtes Königreich (Labour Party 1997-2010)

\begin{tabular}{|c|c|c|}
\hline 1998 & $\begin{array}{l}\text { „New Deal“ für junge Leute } \\
\text { und Langzeitarbeitslose }\end{array}$ & $\begin{array}{l}\text { - Verschärfung der Bezugskriterien von Sozialleistungen } \\
\text { - verbindliche Teilnahme am „New Deal“-Programm }\end{array}$ \\
\hline 1999 & "New Deal“ für Paare & - gemeinsamer Anspruch auf Arbeitslosenunterstützung, wenn Partner ebenfalls auf Job-Suche \\
\hline 2001 & „New Deal“ 25+ & - verbindliche Teilnahme an Arbeitsmarktprogrammen, wenn mindestens 18 der letzten 21 Monate arbeitslos \\
\hline 2002 & $\begin{array}{l}\text { "New Deal“ für Alleinerziehen- } \\
\text { de und für Paare }\end{array}$ & $\begin{array}{l}\text { - verpflichtende Teilnahme an Leistungsanspruchsprüfung für Alleinerziehende } \\
\text { - verbindliche Teilnahme an Leistungsanspruchsprüfung auf Geldleistungen für Arbeitslose für unter 45-Jährige (Paare) }\end{array}$ \\
\hline 2004 & Verschärfung des „New Deal” & - Einstieg in „New Deal“-Programme für junge Leute und 25+ nach drei Monaten in Arbeitslosigkeit \\
\hline
\end{tabular}


Wahlverhalten von Arbeitsmarktinsidern und -outsidern bei nationalen Parlamentswahlen, 2001-2010

Angaben in Prozent

\begin{tabular}{|c|c|c|c|c|c|c|c|}
\hline & \multirow{2}{*}{$\begin{array}{c}\text { Wahljahr } \\
\text { (sozialdem. } \\
\text { geführte Regierung) }\end{array}$} & \multicolumn{3}{|c|}{ Wahlbeteiligung } & \multicolumn{3}{|c|}{$\begin{array}{c}\text { (sozialdemokratische) } \\
\text { Wahlentscheidung }\end{array}$} \\
\hline & & Insider & Outsider & Differenz & Insider & Outsider & Differenz \\
\hline \multirow{3}{*}{ Dänemark } & 2001 (nein) & 93.7 & 91.2 & 2.5 & 27.2 & 33.0 & -5.8 \\
\hline & 2005 (nein) & 93.0 & 92.2 & 0.8 & 24.0 & 33.0 & -9.0 \\
\hline & 2007 (nein) & 92.1 & 92.0 & 0.1 & 23.4 & 28.4 & -5.0 \\
\hline \multirow{3}{*}{ Deutschland } & 2002 (ja) & 84.9 & 83.4 & 1.5 & 33.1 & 38.0 & -4.9 \\
\hline & 2005 (nein) & 84.0 & 78.8 & 5.2 & 32.8 & 35.7 & -2.9 \\
\hline & 2009 (nein) & 81.8 & 78.9 & 2.9 & 27.8 & 27.7 & 0.1 \\
\hline \multirow{3}{*}{ Vereinigtes Königreich } & $2001 \quad$ (ja) & 70.6 & 65.0 & 5.6 & 49.2 & 50.0 & -0.8 \\
\hline & 2005 (ja) & 72.0 & 64.4 & 7.6 & 42.4 & 45.5 & -3.1 \\
\hline & 2010 (nein) & 72.6 & 66.0 & 6.6 & 29.7 & 33.5 & -3.8 \\
\hline
\end{tabular}

Fragen: "Some people don't vote nowadays for one reason or another. Did you vote in the last [country] national election in [month/year]?"/ "Which party did you vote for in that election?"; Sozialdemokratische Parteien: DK = Socialdemokraterne (SD), DE = Sozialdemokratische Partei Deutschlands (SPD), UK = Labour Party $/$ Social Democratic and Labour Party (Labour); Differenz = Differenz zwischen Insidern und Outsidern in Prozentpunkten; nur Personen zwischen 18 und 65 Jahren.

Quelle: Berechnungen der Autoren mit ESS-Daten (gewichtet).

nahmen zum Teil aber bereits deutlich früher umgesetzt wurden (Emmenegger et al. 2012a), kann mit den Daten nicht abschließend geprüft werden, ob tatsächlich ein kausaler Zusammenhang zwischen dem arbeitsmarkt- und sozialpolitischen Wandel und den politischen Einstellungsund Verhaltensmustern der beiden Arbeitsmarktgruppen besteht. Dennoch liefern die Befunde wertvolle Hinweise, die in künftigen Analysen eingehender zu prüfen sind.

Für die Operationalisierung des Insider-/Outsider-Status wurde bereits eine Vielzahl von Vorschlägen unterbreitet (Rueda 2005; Davidsson/Naczyk 2009). Diese Analyse stützt sich auf die Variante von Häusermann/Schwander (2012), wonach Personen zur Gruppe der Outsider gerechnet werden, wenn deren Erwerbssituation aufgrund der spezifischen Merkmalskombination aus Berufsgruppe, Geschlecht und Alter ein signifikant höheres Risiko der Arbeitslosigkeit oder der atypischen Beschäftigung als der Durchschnitt der Bevölkerung ausweist, während Insider keinem überdurchschnittlichen Risiko ausgesetzt sind. ${ }^{5}$

Wenn sich Outsider im Zuge institutioneller Dualisierung von sozialdemokratischen Parteien entfremdet haben, stehen ihnen prinzipiell zwei Möglichkeiten offen, dem durch ihr politisches Verhalten bei Wahlen Ausdruck zu verleihen. So können sie sich entweder durch die Option der Wahlenthaltung vom politischen Prozess zurückziehen (Exit-These), oder ihre Stimme als Ausdruck des politischen Protests an andere (z.B. weiter links orientierte) Parteien vergeben (Voice-These) (Hirschman 1970; Rueda 2005).

Tabelle 1 stellt die prozentualen Anteile der Insider/Outsider dar, die zum Befragungszeitpunkt angaben, an der jeweils letzten nationalen Parlamentswahl teilgenommen und ihre Stimme an eine sozialdemokratische Partei vergeben zu haben. Daraus geht zunächst hervor, dass sich Outsider in allen drei Ländern zu allen Zeitpunkten seltener als Insider an politischen Wahlen beteiligen. Während sich allerdings in Dänemark der ohnehin sehr geringe Abstand zwischen Insidern und Outsidern im Hinblick auf die Wahlbeteiligung zunehmend verringert hat, treten in Deutschland und im Vereinigten Königreich deutlichere Unterschiede hervor. Dabei vergrößert sich der Differenzwert im ersten Fall vor allem zwischen den Bundestagswahlen 2002 und 2005, also direkt im Anschluss an das Inkrafttreten der Hartz-Reformen, und im zweiten Fall zwischen 2001 und 2005, und damit ebenfalls direkt nach Umsetzung der britischen „New Deal“-Politik. In beiden Fällen gehen der Vergrößerung des Abstands zwischen den Arbeitsmarktgruppen also Maßnahmen institutioneller Dualisierung voraus. Zudem trugen sozialdemokratische Parteien in beiden Ländern vor 2005 die Regierungsverantwortung. Insofern erfährt die Exit-These in beiden Ländern einige Unterstützung.

Im Falle Dänemarks ist zu berücksichtigen, dass die zentralen arbeitsmarkt- und sozialpolitischen Reformen

5 Die Einteilung des Berufsstatus erfolgt auf Basis der "Classification of occupations in post-industrial class groups" (Kitschelt/Rehm 2005; Oesch 2006). Die Alterseinteilung bemisst sich an der Unter- bzw. Überschreitung des 40. Lebensjahres. Eine genaue Aufschlüsselung stellen die Autoren auf Anfrage gerne zur Verfügung. 
bereits vor der ersten angeführten Wahl umgesetzt wurden. Erst bei der Parlamentswahl 2001, bei der sich auch die größte Differenz zwischen Insidern und Outsidern einstellt, verlor die Sozialdemokratie ihre Regierungsbeteiligung. Die Verringerung des Abstands zwischen Insidern und Outsidern in den drei folgenden Wahlperioden fällt hingegen in die Amtszeit konservativ-liberaler Regierungen. Diese Entwicklung steht zumindest nicht im Widerspruch zu der Vermutung, das politische Verhalten von Insidern und Outsider sei unmittelbar im Anschluss an institutionelle Dualisierung von zunehmenden Divergenzen geprägt.

Im Hinblick auf das strategische Dilemma sozialdemokratischer Parteien ist allerdings festzustellen, dass sich in allen drei Ländern zu allen Zeitpunkten (außer bei der Wahl zum Deutschen Bundestag 2009) jeweils mehr Outsider als Insider für die Sozialdemokratie entschieden haben. Dennoch ist wenigstens in Deutschland eine Entwicklung zu beobachten, die mit den Vorhersagen der Dilemma- und der Voice-These übereinstimmt. Denn dort verlor die SPD im Verlauf der drei Wahlen zum Deutschen Bundestag unter den Outsidern fast drei Mal mehr Stimmen als unter den Insidern, sodass sich 2009 erstmals mehr Insider als Outsider für die Sozialdemokraten entschieden. Gleichzeitig gewann die Linkspartei in diesem Zeitraum unter den Outsidern immerhin 2,6 Prozentpunkte hinzu (nicht in Tabelle ausgewiesen). In den beiden anderen Ländern vergrößerte sich der Abstand zwischen Insidern und Outsidern im Zeitverlauf tendenziell zwar auch, jedoch in entgegengesetzter Richtung. Hier sind es vor allem Insider, die sich in größerem Maße als Outsider von der Sozialdemokratie abgewendet haben. Die VoiceThese erfährt aufgrund der angeführten Befunde daher allenfalls für die Bundesrepublik einige Unterstützung.

Mögliche Erklärungen für diese von den theoretischen Erwartungen abweichenden Befunde sollten vor allem im zeitlichen Abstand zur Umsetzung institutioneller Dualisierung und der zurückliegenden Regierungsbeteiligung sozialdemokratischer Parteien (Lewis-Beck/Paldam 2000; Duch/ Stevenson 2008) sowie dem jeweiligen Wahl- und Parteiensystem gesucht werden (Decker 1999). So trugen im vergangenen Jahrzehnt in Dänemark hauptsächlich konservative und liberale Parteien Regierungsverantwortung, während institutionelle Dualisierungsmaßnahmen (etwa in Gestalt der Hartz-Reformen) von der sozialdemokratisch geführten Regierung in Deutschland von den Daten noch eingefangen werden. Auch im Vereinigten Königreich regierte in der letzten Dekade mit der Labour Party die Sozialdemokratie. Das dortige Mehrheitswahlsystem erschwert jedoch die Etablierung einer weiteren linken Partei, die fortan die arbeitsmarktund sozialpolitischen Interessen der Outsider vertreten könnte, wie es im deutschen Verhältniswahlsystem der Linken gelungen ist. Deutschen Outsidern eröffnet sich aufgrund des Wahl- und Parteiensystems so überhaupt erst die Möglichkeit, von der Voice-Option Gebrauch zu machen, während im Vereinigten Königreich für Outsider die Exit-Option naheliegender ist. Diese Zusammenhänge sollten künftig eingehender empirisch geprüft werden.

\section{Fazit}

In der vergleichenden Sozialstaatsforschung werden Arbeitsmarktreformpolitiken, wie sie in Deutschland die Hartz-Gesetzgebung implementierte, als ein Prozess der Dualisierung beschrieben. Der Beitrag griff das Konzept auf, argumentierte jedoch, dass nicht erst die Hartz-Reformen die Aufspaltung in In- und Outsider schufen. Vielmehr entstanden bereits zuvor mit dem Wandel der Einkommensverteilung und der Ausrichtung sozialdemokratischer Parteien die Voraussetzungen für politische Reformen, die eine institutionelle Dualisierung brachten. Es bestätigte sich, dass sich bereits vor den Hartz-Reformen die materielle Lage zwischen unteren und mittleren Schichten zunehmend auseinanderentwickelte (siehe auch den Beitrag von Rudolph in diesem Heft). Während Haushalte mit geringen Einkommen, in denen sich vor allem Arbeitsmarkt-Outsider wiederfinden, im hier betrachteten Zeitraum von 1980 bis 2005 Einbußen bei den am Arbeitsmarkt erzielten Einkommen hinnehmen mussten, war die relative Einkommensposition der mittleren Einkommensgruppen stabil, was darauf verweist, dass die Arbeitsmarktchancen der gesellschaftlichen Mitte kaum in Mitleidenschaft gezogen sind. Somit bot bereits die materielle Lage wenig Anreize für arbeitsmarkt- und verteilungspolitische Interessenkoalitionen zwischen beiden Gruppen. Insbesondere die politisch einflussreiche Mittelschicht hatte kaum Anlass, einer Politik der Dualisierung, die soziale Risiken nach unten verteilt, entgegenzutreten.

Auch die zurückliegende Entwicklung der ideologischen Ausrichtung sozialdemokratischer Parteien bereitete die Basis für eine Dualisierungspolitik, da die Verlagerung der Links-Rechts-Position der Sozialdemokratie hin zur Mitte anzeigt, dass sie sich zunehmend an Personen orientierte, die eher zu den Arbeitsmarkt-Insidern zählen. Mit der Bewegung in die Mitte verringerten sozialdemokratische Parteien den Abstand zum wahlentscheidenden Median-Wähler.

So waren es dann auch vor allem sozialdemokratisch geführte Regierungen, die in den 1990er und 2000er Jahren zentrale arbeitsmarktpolitische Reformen in den betrachteten Ländern umsetzten - mit Folgen für das politische Verhalten der Arbeitsmarktinsider bzw. -outsider. Insbesondere bei Wahlen, die im unmittelbaren Anschluss an institutionelle Dualisierungsmaßnahmen stattfanden, taten sich zunehmend Lücken in der Wahlbeteiligung zwischen Insidern und Outsidern auf. Vor allem bei den jeweiligen Parlamentswahlen 2005 in Deutschland und Großbritannien verringerte sich die Wahlbeteiligung unter den Outsidern unmittelbar im Anschluss an die Einführung der Hartz-Gesetze bzw. der „New Deal“Politik. Dies deutet darauf hin, dass sich die institutionelle Dualisierung im Zuge der Arbeitsmarktreformen tatsächlich in dualisierte politische Verhaltensweisen umgesetzt hat.

Hinsichtlich der Wahlentscheidung konnte - abgesehen von Deutschland - jedoch nicht gezeigt werden, dass Outsider ihre Stimme gegenüber Insidern seltener an sozialdemokratische Parteien vergaben. Im Gegenteil waren es in 
Dänemark und im Vereinigten Königreich eher die Insider, die sich stärker von sozialdemokratischen Parteien abgewendet haben.

Der vorliegende Beitrag soll einer verkürzten Debatte vorbeugen, die die Hartz-Gesetze als alleinige Ursache von Dualisierung thematisiert. Wir zeigen, dass vielmehr der langfristige Wandel der Einkommensstrukturen, daraus resultierende politische Interessen und der Wandel der parteipolitischen Programmatik Grundlagen für eine Arbeitsmarktreform waren, die von einer dualen (weil für unterschiedliche Gruppen unterschiedliche Regelungen vorsehenden) institutionellen Logik geprägt ist. Einerseits wird es Outsidern erschwert, ihre Arbeitsmarkt- und Verteilungsinteressen politisch durchzusetzen, da die Sozialdemokratie sich in die Mitte des Links-Rechts-Spektrums bewegt und so eher Insider-Positionen in ihre Programmatik aufnimmt. Auf der anderen Seite sehen sich auch sozialdemokratische Parteien erheblichen Problemen gegenüber. Sie treffen in der Mitte auf parteipolitische Konkurrenz in Gestalt der Christdemokratie, die ebenfalls eine Politik der Dualisierung befürwortet und praktiziert, um die Interessen von Facharbeitern, technischen Berufen und im Dienstleistungssektor tätigen Professionellen mit in der Regel konservativeren politischen Orientierungen zu bedienen (Iversen/Soskice 2009). Daran anschließende Fragen, wie denn Arbeitsmarktreformen ausgesehen hätten unter anderen Regierungen, lassen sich hier nicht vertiefen. Analysen lassen jedoch erwarten, dass von christdemokratischer Mehrheit geprägte Reformen eine noch weitergehende Re-Kommodifizierung und Deregulierung gebracht hätten (Swank 2013).

Dass Median-Wähler und sozialdemokratische Parteien in Zukunft wieder stärker nach links tendieren, schließen die hier verwendeten Argumente und Daten keineswegs aus. Die Politik der Deregulierung der Arbeitsverhältnisse und der De-Kommodifizierung durch ein gesenktes Niveau sozialer Sicherung und verschärfter Zumutbarkeitsregeln erreicht durchaus auch Insider, da der antizipierte Arbeitsplatzverlust heute nach den Reformen ein größeres Drohpotenzial hat (Knuth 2011). Das erhöht die Chance auf eine neue Brückenbildung zwischen Insidern und Outsidern.

\section{LITERATUR}

Arndt, C. (2013): The electoral consequences of third way welfare state reforms. Social democracy's transformation and its political costs, Amsterdam Bispinck, R./Bosch, G. (2008): Auflösung des deutschen Tarifmodells?, in: Wirtschaftsdienst 88 (1), S. 7-27

Budge, I./Klingemann, H.-D./Volkens, A./Bara, J./Tanenbaum, E. (Hrsg.) (2001): Mapping policy preferences. Estimates for parties, electors, and governments 1945-1998, Oxford

Dallinger, U. (2013): Economic openness and domestic demand for social protection: A multi-level analysis of social security preferences between 1990 and 2006, in: Comparative Sociology 12 (5), S. 585-616

Davidsson, J./Naczyk, M. (2009): The ins and outs of dualisation. A literature review, in: Working Papers on the Reconciliation of Work and Welfare in Europe 02/2009, S. 1-44

Decker, F. (1999): Parteien und Parteiensysteme im Wandel, in: Zeitschrift für Parlamentsfragen 30 (2), S. 19-61

Diewald, M./Sill, S. (2005): Mehr Risiken, mehr Chancen? Trends in der Arbeitsmarktmobilität seit Mitte der 1980er Jahre, in: Struck, O./Köhler, C. (Hrsg.): Beschäftigungsstabilität im Wandel? Empirische Befunde und theoretische Erklärungen für West- und Ostdeutschland, München, S. 39-62 Dingeldey, I. (2010): Agenda 2010. Dualisierung der Arbeitsmarktpolitik, in: Aus Politik und Zeitgeschichte (48), S. 18-25

Downs, A. (1957): An economic theory of democracy, New York

Duch, R./Stevenson, R. (2008): The economic vote. How political and economic institutions condition election results, Cambridge

Emmenegger, P./Häusermann, S./Palier, B./Seeleib-Kaiser, M. (2012a): How we grow unequal, in: Emmenegger et al. (2012c), a.a.O., S. 3-26
Emmenegger, P./Häusermann, S./Palier, B./Seeleib-Kaiser, M. (2012b): How rich countries cope with deindustrialization, in: Emmenegger et al. (2012c), a.a. O., S. $304-320$

Emmenegger, P./Häusermann, S./Palier, B./Seeleib-Kaiser, M. (Hrsg.) (2012c): The age of dualization. The changing face of inequality in deindustrializing societies, Oxford

Erlinghagen, M. (2010): Zunehmende Angst vor Jobverlust trotz gleichbleibender Beschäftigungsstabilität, in: Informationsdienst Soziale Indikatoren (44), S. 1-4 Esping-Andersen, G. (1990): The three worlds of welfare capitalism, Princeton Esping-Andersen, G. (2009): Changing classes. Stratification and mobility in post-industrial societies, London

Giesecke, J./Heisig, J. (2010): Destabilisierung und Destandardisierung, aber für wen? Die Entwicklung der westdeutschen Arbeitsplatzmobilität seit 1984, in: Kölner Zeitschrift für Soziologie und Sozialpsychologie 62 (3), S. 403-435 Gießelmann, M. (2009): Arbeitsmarktpolitischer Wandel in Deutschland seit 1991 und das Working Poor-Problem. Einsteiger als Verlierer des Reformprozesses?, in: Zeitschrift für Soziologie 38 (3), S. 215-238

Giger, N./Rosset, J./Bernauer, J. (2012): The poor political representation of the poor in a comparative perspective, in: Representation 48 (1), S. 47-61 Harrison, B./Bluestone, B. (1988): The great u-turn, New York

Häusermann, S./Schwander, H. (2012): Varieties of dualization. Labor market segmentation and insider-outsider divides across regimes, in: Emmenegger et al. (2012c), a.a.O., S. 27-51

Hirschmann, A. (1970): Exit, voice, and loyalty. Responses to decline in firms, organizations, and states, Cambridge

Immervoll, H./Richardson, L. (2013): Redistributive policy in Europe and the United States. Is the Great Recession a game changer for working age families?, OECD Working Papers (150), DELSA/ELSA/WD/SEM(2013)6

Iversen, T./Soskice, D. (2009): Dualism and political coalitions. Inclusionary versus exclusionary reforms in an age of rising inequality. Paper presented at the Annual Meeting of the APSA, Toronto, September

Keller, B./Schulz, S./Seifert, H. (2012): Entwicklungen und Strukturmerkmale atypisch Beschäftigter in Deutschland bis 2010, WSI-Diskussionspapier (182), S. $1-25$

Kim, H./Fording, R. (1998): Voter ideology in western democracies, 1946-1989, in: European Journal of Political Research 33 (1), S. 73-97

Kim, H./Fording, R. (2003): Voter ideology in western democracies. An update, in: European Journal of Political Research 42 (1), S. 95-105

Kitschelt, H./Rehm, P. (2005): Work, family and politics. Foundations of electoral partisan alignments in postindustrial democracies. Paper prepared for delivery at the annual meeting of the HASPA, Washington, DC, September

Knuth, M. (2011): Widersprüchliche Dynamiken im deutschen Arbeitsmarkt, in: WSI-Mitteilungen 64 (11), S. 580-587, http://www.boeckler.de/wsi_38168_38177. htm

Lengfeld, H./Hirschle, J. (2009): Die Angst der Mittelschicht vor dem sozialen Abstieg. Eine Längsschnittanalyse 1984-2007, in: Zeitschrift für Soziologie 38 (5), S. 379-398

Lewis-Beck, M./Paldam, M. (2000): Economic voting. An introduction, in: Elec toral Studies $19(2-3)$, S. $113-121$

Lindvall, J./Rueda, D. (2012): Insider-outsider politics. Party strategies and political behavior in Sweden, in: Emmenegger et al. (2012c), a.a.O., S. 277-303

Lipset, S./Rokkan, S. (Hrsg.) (1967): Party sytems and voter alignments. Crossnational perspectives, New York

Lohmann, H. (2010): Armut von Erwerbstätigen im europäischen Vergleich: Erwerbseinkommen und Umverteilung, in: Kölner Zeitschrift für Soziologie und Sozialpsychologie 62 (1), S. 1-30

Marx, P. (2012): Labour market dualisation in France. Assessing different explanatory approaches, in: European Societies 14 (5), S. 704-726

Meltzer, A./Richard, S. (1981): A rational theory of the size of government, in: Journal of Political Economy 89 (5), S. 914-927

Nelson, S./Way, C. (2007): Party crashers. The determinants of left party ideological shifts in wealthy democracies. Paper presented at the annual meeting of the MPSA, Chicago, April 12

Organisation for Economic Co-operation and Development (OECD) (2011): Divided we stand. Why inequality keeps rising, Paris

Oesch, D. (2006): Redrawing the Class Map. Stratification and Institutions in Germany, Britain, Sweden, and Switzerland, London

Palier, B./Thelen, K. (2010): Institutionalizing dualism. Complementarities and Change in France and Germany, in: Politics and Society 38 (1), S. 119-148

Pontusson, J. (1995): Explaining the decline of european social democracy: The role of structural economic change, in: World Politics 47 (4), S. 495-533 
Pontusson, J./Rueda, D. (2010): The politics of inequality. Voter mobilization and left parties in advanced industrial states, in: Comparative Political Studies 43 (6), S. $675-705$

Pontusson, J./Rueda, D./Way, C. (2002): Comparative political economy of wage distribution. The role of partisanship and labour market institutions, in: British Journal of Political Science 32 (2), S. 281-308

Rueda, D. (2005): Insider-outsider politics in industrialized democracies: The challenge to social democratic parties, in: American Political Science Review 99 (1), S. $61-74$

Rueda, D. (2007): Social democracy inside out. Partisanship and labor market policy in advanced industrialized democracies, Oxford

Schoen, H. (2005): Soziologische Ansätze in der empirischen Wahlforschung, in: Falter, J./Schoen, H. (Hrsg.): Handbuch Wahlforschung, Wiesbaden Swank D. (2013): Party government, institutions, and social protection in the age of austerity, in: Armingeon, K. (Hrsg.): Staatstätigkeit, Parteien und Demokratie. Festschrift für M. G. Schmidt, Wiesbaden

Thelen, K. (2012): Varieties of capitalism. Trajectories of liberalization and the new politics of social solidarity, in: Annual Review of Political Science 15 (1), S. $137-159$

Verba, S./Schlozman, K./Brady, H. (1995): Voice and equality. Civic voluntarism in American politics, Cambridge
AUTOREN

URSULA DALLINGER, Dr., Professorin für Soziologie und Sozialpolitik an der UniversitätTrier. Arbeitsschwerpunkte: Vergleichende Forschung zu Einkommensungleichheit und sozialstaatlicher Umverteilung, sozialpolitische Einstellungen im internationalen Vergleich.

dallinger@uni-trier.de

SEBASTIAN FÜCKEL, Wissenschaftlicher Mitarbeiter an der Professur für Soziologie und Sozialpolitik der UniversitätTrier. Arbeitsschwerpunkte: Politische Soziologie, empirische Wahlforschung, soziale Ungleichheit und vergleichende Sozialstrukturanalyse.

fueckel@uni-trier.de 